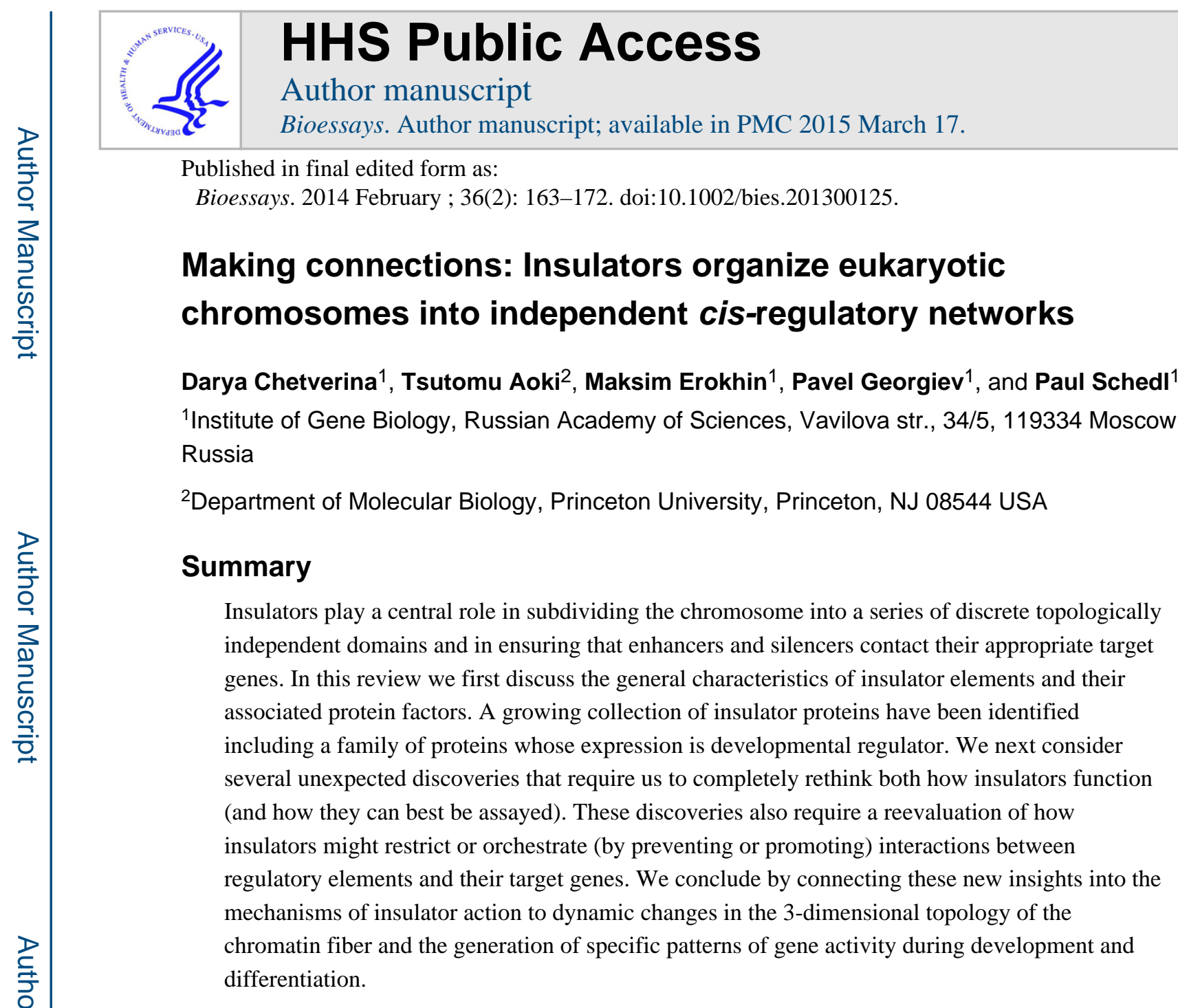

\title{
Introduction
}

The development of multicellular eukaryotes depends upon the proper execution of complex cell- and tissue-specific programs of gene activity. While activity can be modulated at many different levels, one point that is especially critical is at the level of transcription. The proper programming of transcriptional activity pivots on ensuring that regulatory elements, enhancers and silencers, control the activity of their "designated" target genes, without inappropriately affecting other differentially regulated genes in the surrounding neighborhood. There is a growing body of evidence that correctly matching enhancers and silencers with their target genes depends not only upon the linear arrangement of these elements along the chromosome, but also on how they are organized in 3-dimensional space.

Though not fully understood at the time, one of the first hints that regulation is intimately connected to the 3-dimensional organization of the chromatin fiber came from studies on the Drosophila Notch locus by Welshon and Keppy in the 1970's. They identified a mutation, $f a^{\text {strawberry }}\left[f a^{s t b}\right]$, located just upstream of the Notch promoter which had two significant properties. First, the mutation induced a "chromosomal" position effect that interfered with Notch expression in the eye. Second, in salivary gland polytene chromosomes it resulted in the fusion of two adjacent bands, 3C7, which contains Notch, and 3C6 [1]. Subsequent work 
demonstrated that $f a^{s t b}$ is a small deletion that removes a special $c i s$-acting element, a chromatin domain boundary or insulator, that functions to organize 3C7 and 3C6 into topologically independent domains [2]. In this review, we focus on the properties of this special class of elements, and discuss their role in determining the 3-dimensional organization of the chromosome and in defining units of genetic activity.

Insulators were first identified in Drosophila; however, they have since been found in many other eukaryotes, ranging from yeast to humans [see 3, 4]. While functional studies are limited to only a hundred or so examples, genome-wide ChIPs for known insulator proteins indicate that they are ubiquitous components of the genome. For example, human CTCF insulator protein localizes to $\sim 13,000-30,000$ sites depending upon the cell line [5,6], while Drosophila CTCF localizes to $\sim 2,500$ sites [7].

Though a handful of insulators have been discovered on the basis of their mutant phenotypes, most have been identified using two assays, enhancer blocking and barrier activity (Fig. 1). These assays were based on the idea that insulators define independent units of gene activity. In the enhancer-blocking assay, a putative insulator is interposed between an enhancer and the target gene promoter. In this position an insulator blocks or attenuates enhancer action; however, it is unable to affect enhancer action when the order is reversed and the enhancer, not the insulator, is closer to the promoter (Fig. 1). In barrier assay, insulators bracketing a reporter protect against the encroachment of heterochromatic repression from the chromatin flanking the transgene insertion site (Fig. 1). In addition, most insulators are able to block silencing by Polycomb response elements (PREs) when placed between the PRE and the target gene.

In the sections that follow, we first review the general properties of insulators and their protein constituents. Here we highlight the growing collection of proteins implicated in insulator activity, including the discovery of a factor whose activity is developmentally restricted. We then focus on several recent mechanistic insights that require us to rethink how insulators function and reevaluate how insulators might restrict or orchestrate interactions between regulatory elements and their target genes. In the concluding section we explore how insulators coordinate gene activity in one of the classic multigene loci of Drosophila, the bithorax complex, and then relate insulator activity to recent insights into the topological organization of eukaryotic chromosomes that have come from $3 \mathrm{C}$ (Chromatin Conformation Capture) experiments.

\section{Properties of insulators}

\section{Insulators are assembled by combining functionally redundant cis-acting sequences}

The fly and vertebrate insulators that have been characterized typically range in length from several hundred bp to more than a $\mathrm{kb}$ and encompass one or more nuclease hypersensitive regions in chromatin. Within these elements are multiple recognition sequences for proteins that confer insulator activity. While CTCF is thought to be the only vertebrate insulator protein [8], six sequence specific factors $\{\mathrm{Su}(\mathrm{Hw})[9,10], \mathrm{dCTCF}[11]$, Zeste-white 5 (Zw5) [12], GAGA factor [GAF] [13,14], boundary element associated factor (BEAF)[15] and the 
tripartite Elba complex [16] \} have been identified in flies, and it is clear from ongoing work that this list is far from complete.

Of the known insulators, $s u(H w)$ from the gypsy transposon has the simplest sequence organization [17]. It contains twelve $\mathrm{Su}(\mathrm{Hw})$ binding sites. While full insulating activity doesn't require all twelve sites, activity is weaken when the number is reduced below six [17]. Endogenous fly insulators differ from $s u(H w)$ in that they don't consist of reiterated sites for only a single protein. For example, an endogenous " $s u(H w)$ " insulator downstream of the yellow gene, 1A2, has only two $\mathrm{Su}(\mathrm{Hw})$ sites $[18,19]$ and full activity requires additional unidentified sequences and factors [20]. On the other hand, endogenous insulators resemble $s u(H w)$ in that they are assembled from a series of functionally redundant subelements that on their own are capable of conferring partial or even full activity. For example, the insulator activity of scs from the $87 \mathrm{~A} 7$ heat shock locus can be reconstituted by multimerizing short sub-elements that are unrelated in sequence [12]. Of course, the fact that insulators are ensembles of functionally redundant sub-elements complicates genetic analysis as disruption of a single or even several sub-elements may have only limited effects on boundary activity. For example, mutations in 5 of the 6 GAF binding sites in the bithorax complex (BX-C) Fab-7 insulator have only a modest effect on activity [13]. Likewise, inactivation of the CTCF sites of the BX-C Fab-8 insulator only partly reduces its enhancerblocking activity [11]. A further indication of functional redundancy comes from genome wide ChIP experiments [21-26]. Thus, for the homie insulator downstream of the evenskipped (eve) gene [27] ModEncode lists $\mathrm{Su}(\mathrm{Hw}) \mathrm{dCTCF}, \mathrm{GAF}$ and BEAF [25]. Also given the complexity of the cis-elements and trans-acting factors associated with typical insulators, it is not altogether surprising that RNAi knockdowns of insulator proteins in flies often show only minimal effects on insulator activity (e.g., the spread of Polycomb silencing $[22,26])$.

\section{Multiple trans-acting factors have insulator activity}

Four of the sequence specific insulator proteins that have been identified in flies, $\mathrm{Su}(\mathrm{Hw})$, $\mathrm{Zw} 5, \mathrm{dCTCF}$ and GAF are $\mathrm{C} 2 \mathrm{H} 2$-type zinc finger proteins. The most thoroughly studied, $\mathrm{Su}(\mathrm{Hw})$, has twelve $\mathrm{C} 2 \mathrm{H} 2$-type zinc fingers, acidic $\mathrm{N}$ and $\mathrm{C}$ terminal domains and a leucine zipper motif [28]. While the zinc fingers mediate DNA binding, insulator activity depends upon recruiting partners like Mod(mdg4)-67.2 [29-31]) and Centrosomal Protein 190 (CP190) [32]. The Mod(mdg4)-67.2 protein contains an the N-terminal insect-specific variant of the BTB domain that mediates self-association [33,34]. While CP190 also has a BTB domain, it belongs to a more broadly conserved class that exists as obligate homodimmers [34]. A third partner, $\mathrm{E}(\mathrm{y}) 2$, doesn't function in enhancer blocking, but is important for preventing the spread of silencing [35].

The vertebrate and fly CTCF proteins both contain 11 zinc fingers and have a similar spectrum of recognition sequences [36]. CTCF was first implicated in the insulator activity of the chicken $\beta$-globlin locus cHS4 element [37], and has since been shown to be important for the activity of a growing collection of vertebrate insulators [36]. CTCF requires the cohesin complex (SA2, Rad21, SMC1/3) and a region in its C-terminus interacts directly with the cohesin SA2 subunit [38]. CTCF is believed to provide the sequence specificity, 
while cohesin functions to link two distant DNAs together. In flies, the CP190 protein binds to CTCF, and is required for enhancer blocking by CTCF dependent insulators [38-40].

Unlike $\mathrm{Su}(\mathrm{Hw})$ or $\mathrm{CTCF}$, GAF has only a single $\mathrm{Zn}$ finger located in its C-terminal half. The N-terminal half has a BTB domain that forms oligomeres, and mediates interactions with other BTB proteins [41-3]. GAF has been implicated in the insulator activity of Fab-7 [13] and the Antennapedia (Ant) boundary $S F 1$ [14] and is found associated with many putative insulators (along with CP190) in ChIP experiments [25]. However, GAF also has other activities including: transcription activation [44, 45], nucleosome positioning [46,47], Pol II pausing [48], and Polycomb Response Element (PRE) dependent silencing [49]. While it is not understood how GAF carries out these different functions, one idea is that each depends upon a different collection of partners.

Since vertebrate CTCF and the various fly insulator proteins are expressed throughout development and can confer insulator activity in a wide range of tissues and cell types, it was assumed that insulators define rather static chromatin domains that are generally invariant from one cell type to the next (with the notable exception of $I g f 2 / H 19$ locus where the domain organization differs depending upon whether the chromosome is from the mother or father [50, 51]). However, a number of lines of evidence now suggest that domains are organized and reorganized in different patterns during development and differentiation, thereby changing units of genetic activity. One comes from the discovery of a tripartite Drosophila insulator complex, called Elba, whose activity is developmentally restricted. Elba subunits, Elba1 and Elba2 [16], share a conserved C-terminal 'BEN" domain that mediates DNA binding [52, 53]. The third subunit, Elba3 is responsible for forming the tripartite complex. All three Elba subunits are required both for DNA binding in vitro and insulator function in vivo [16]. Elba activity is developmentally restricted because midblastula transition genes encode Elba1 and Elba3. Flies have two other BEN DNA binding proteins. One is called Insensitive (Insv) and is involved neurogenesis and Notch signaling $[53,54]$, while the other is an as yet uncharacterized $\operatorname{Mod}(\operatorname{mdg} 4)$ isoform.

Interestingly, mammalian genomes also have multiple BEN domain genes. Elba1 is most closely related to mammalian BEND7, while Elba2 is most closely related to BEND6, a protein recently shown to antagonize Notch signaling in self-renewing mouse neural stem cells [55]. The mammalian Insv orthologs are BEND9/hNAC2 and BEND8/hNAC1, which have been implicated in stem cell pluripotency and carcinogensis [56,57]. It seems likely that several of the mammalian BEN proteins will have tissue or stage specific insulator functions just like the fly BEN proteins. In this case, CTCF would not be the only mammalian DNA binding insulator protein. Other findings support this idea. For example, Srivastava et al [58] have recently identified a series of insulators in the intergenic regions of the mouse Hox clusters. These Hox insulators map to nucleosome free regions and are bound by the mammalian GAF protein, Th-POK, and not CTCF.

\section{Are insulators functionally autonomous or non-autonomous elements?}

One question that it is central to understanding both how insulators act and how they impact gene regulation is whether they function autonomously or non-autonomously. When insulators were first discovered it was assumed that they were functionally autonomous 
elements and that their activities were not only context independent but also did not involve or require any sorts of interactions with other insulators. This assumption was reflected in the design of the original assays. For example, in the enhancer-blocking assay only a single insulator (interposed between the enhancer and the reporter) was included in the transgene reporter (Fig. 1). However, there is now abundant evidence that this assumption (at least for flies) is entirely incorrect. Insulators don't function autonomously; instead, their activity not only depends upon but is also determined by the identity of other insulators in their neighborhood. This non-autonomy is an inherent feature of the insulator:insulator interactions (or interactions with other DNA elements) that are responsible not only for organizing the chromosome into a series topological loops but also for many of the important regulatory activities of this class of elements. Of course, if insulators don't function autonomously, assays that assume autonomous, context independent activity can't be used to test whether candidate elements identified in, for example, genome-wide ChIPs are insulators $[25,26]$. In the sections below we discuss the evidence for non-autonomy and explore how this affects the process of domain definition and gene regulation.

Insulators can be bypassed-The first indication of non-autonomy was the discovery of "insulator bypass." While a single $s u(\mathrm{Hw})$ insulator placed between an enhancer and reporter blocks the enhancer, blocking activity is lost when two $\mathrm{su}(\mathrm{Hw})$ insulators are interposed between the enhancer and the reporter [59,60]. As illustrated in Fig. 2, it is thought that pairing of the two $s u(H w)$ insulators brings the enhancer in close proximity to the promoter, enabling it to bypass the insulators. Bypass has also been observed when a PRE silencer is substituted for the enhancer [61]. Insulator bypass has since been demonstrated for a large number of fly insulators and appears to be a common property of this class of DNA elements [62-65]. (There is evidence suggesting that vertebrate CTCF insulators are bypassed as well [66]).

Insulator-dependent pairing interactions-Consistent with a mechanism dependent upon insulator pairing, 3C experiments showed that the insulators flanking the 87A7 heat shock locus, scs and scs', are in contact with each other in vivo [15,67]. Moreover, contact is mediated, at least in part, by physical interactions between Zw5 bound to scs and BEAF bound to scs'. Contacts between vertebrate CTCF insulators have also been demonstrated [68-70], and as noted above are thought to be mediated by cohesin [71-3]. In addition to interactions with neighboring insulators, insulator pairing can take place over very long distances (> $1 \mathrm{Mb}$ ), between elements inserted on different chromosomal arms or even on different chromosomes. The first evidence for insulator dependent long distance interactions came from genetic assays with transgenes located at distant sites containing the BX-C Mcp [74,75] or the gypsy su(Hw) insulators [76]. Other insulators, like Fab-7 and homie, are also able to mediate long distance genetic interactions [27,77]. Moreover, recent studies show the genetic interactions in these experiments involve direct physical contact between insulators in the distant transgene inserts [78].

Insulators compete to define regulatory domains-Direct evidence that the activity of an insulator depends upon the identity of potential partners in the neighborhood comes from a series of insulator competition assays. In one such assay (see the domain definition 
assay in Fig. 3), an insulator, Fab-7, is interposed between the fushi-tarazu stripe [UPS] and neurogenic [NE] enhancers and hsp70-lacZ, which is one of the two reporters in the transgene [79]. In this configuration, the other reporter, white, and the ftz enhancers are together in one regulatory domain. The UPS enhancer drives white expression in a stripe pattern in early embryos, while the NE enhancer drives white in the CNS during midembryogenesis. In contrast, the hsp70-lacZ reporter is in a separate domain defined by Fab-7 and scs' that is insulated from the ftz enhancers. The introduction of a competing insulator, $s u(H w)$, between white and the enhancers redefines the domain organization. In the new configuration, the $f t z$ enhancers, Fab-7, and $h s p 70-l a c Z$ are in the same regulatory domain, and the enhancers bypass Fab-7, activating hsp70-lacZ. In contrast, the enhancers don't activate white. Interestingly, bypassing Fab-7 depends upon both the developmental stage and the insertion site. In a subset of the inserts $\mathrm{Fab}-7$ "regains" insulator activity during mid-embryogenesis and blocks the NE enhancer from activating hsp70-lacZ (Fig. 3).

Pairing interactions are both specific and directional-Insulator competition experiments like the domain definition assay not only show that insulators have to function with partners but also that some partners make much better matches than others. Further evidence for the importance of properly matching partners comes from the bypass assay. For example, no bypass is observed when $s u(H w)$ is paired with $s c s, F a b-7$ or $S F 1$, while bypass is observed when $F a b-7$ or scs are paired with themselves [64,66, 80, 81]. Though the precise basis for specificity is not yet understood, it clearly depends upon the factors associated with each insulator.

Evidence supporting this conclusion comes from experiments in which multimerized binding sites for $\mathrm{Su}(\mathrm{Hw}), \mathrm{CTCF}$ or $\mathrm{Zw} 5$ were used in place of insulators. While "homologous" binding site combinations \{e.g., 4x su(Hw) paired with 4x su(Hw) \} supported bypass, "heterologous" combinations \{e.g., 4x su(Hw) paired with 4x CTCF did not [66]. Two other findings support the notion that insulator:insulator interactions are highly specific. First, composite multimers containing sites for two different insulator proteins $\{4 \mathrm{x}$ su(Hw):4x CTCF paired with $4 \mathrm{x}$ su(Hw):4x CTCF $\}$ support bypass. Second, the bypass mediated by these composite sites is orientation dependent [65]. As illustrated in Fig. 4, this is thought to reflect the topology of the loop formed between the two sets of binding sites. In one case, the loop brings the enhancer and promoter in close proximity, while in the other the enhancer and promoter are separated by the loop. Orientation dependence has also been observed for endogenous insulators like Mcp, scs, and scs' $[65,82-4]$ and, as is the case for the composite multimers, this dependence implies that loop formation is mediated by quite specific interactions between the factors associated with the paired insulators.

\section{Insulators mediate enhancer-promoter interactions}

One other activity that is relevant to the functioning of insulators in both 3-dimensional topology and gene regulation are interactions between insulators and promoters. Such physical interactions were first detected by $3 \mathrm{C}$ in the mouse $\beta$-globin locus between the two insulators at the borders, the enhancers in the Locus Control Region (LCR) and the promoter of the active $\beta$-globin gene [85]. A similar clustering of insulators, enhancers and promoters 
is observed in the imprinted $I g f 2 / H I 9$ locus and both clustering and $I g f 2$ expression depend upon cohesin $[71,72,86]$. The existence of multiple long-range interactions between promoters and CTCF-bound sites in vertebrates was recently confirmed [66].

In Drosophila evidence for insulator-promoter interactions comes from studies on the yellow $1 \mathrm{~A} 2$ and the white WARI insulators [87]. When placed downstream of a reporter and binding sites for the yeast Gal4 transcription factor, both of these insulators stimulate Gal4 activation of the reporter. Unlike insulator bypass, this stimulation is independent of insulator orientation. Fujioka, Sun and Jaynes (pers. comm.) recently discovered an even more striking example of insulator dependent enhancer-promoter interactions in the eve locus. As noted above, the homie insulator is downstream of eve between the stripe enhancers 1, 4, 5, and 6 and the neighboring TER94 gene [27]. Deletion experiments show that in addition to ensuring that eve and TER94 function autonomously, homie is also require by these four stripe enhancers to activate eve stripe expression. In contrast, homie isn't important for the activity of the three stripe-enhancers $(2,3$, and 7$)$ upstream of eve. One potential mechanism for insulator dependent enhancer-promoter interaction is suggested by the finding that mammalian CTCF interacts with a component of TAFIID complex, TAF3 [88]. There is yet another connection between insulators and promoters. Promoters have been found associated with several of the known insulators in flies including scs, scs', IdefixU3, and $F a^{s w b}$, while several promoters (eve, white and stalled polymerases in BX-C) have been found to have insulator activity.

\section{Insulators organize the chromosome into cis-regulatory networks}

The novel and unexpected properties of insulators (non-autonomy, bypass, enhancerpromoter interactions, and developmental regulation) together with new insights into the topological organization of the genome that have come from $3 \mathrm{C}$ experiments require a rethinking of how these elements function in chromosome organization and gene regulation. In this section, we focus first on $\mathrm{BX}-\mathrm{C}$, as these novel insulator activities are central to the functioning of this complex. Here, we present a semi-speculative discussion of how insulators organize and control BX-C activity. We then attempt to relate these ideas to new discoveries about genome topology.

\section{Insulator and the functioning of the fly Bithorax complex}

The three homeotic genes, $U b x, a b d-A$ and $A b d-B$ in BX-C are controlled by a large $>300 \mathrm{~kb}$ cis-regulatory domain that is subdivided into 9 functionally autonomous units [89). Two of these, $a b x / b x$ and $b x d / p b x$ define the $U b x$ Transcriptional Associated Regulatory Domain (TARD). Three $c i s$-regulatory domains, $i a b-2, i a b-3$ and $i a b-4$ comprise the $a b d-A$ TARD, while the $A b d-B$ TARD has four domains, $i a b-5, i a b-6, i a b-7$ and $i a b-8$ (Fig. 5).

How these cis-regulatory domains and their associated insulators function in coordinating homeotic gene activity is best understood for the $A b d-B$ TARD. As shown in Fig. 5, the three insulators in the $A b d-B$ TARD, Fab-6, Fab-7 and Fab-8, are located between the $i a b-5, i a b-6, i a b-7$, and $i a b-8$ cis-regulatory domains, respectively. A fourth insulator, $M c p$, separates the $A b d-B$ TARD from the adjacent $a b d-A$ TARD. Each domain in the $A b d-B$ TARD controls $A b d-B$ expression, and thus parasegment identity, in a specific parasegment: 
$i a b-5$ specifies PS10 identity, $i a b-6$ PS11, $i a b-7$ PS12 and $i a b-8$ PS13. The four Abd-B cisregulatory domains are sequentially activated in successive posterior parasegments. For example, in PS10, $i a b-5$ is turned on and regulates $A b d-B$ expression, while $i a b-6,7$ and 8 are silenced by the recruitment of Polycomb complexes to PREs located in each domain (Fig. 5B). In the next parasegment, PS11, both $i a b-5$ and $i a b-6$ are turned on, while $i a b-7$ and $i a b-8$ remain off; however, $A b d-B$ expression is controlled by the (activated) domain that is closest to $A b d-B$, in this case $i a b-6$ (Fig. 5B).

One activity of the $A b d-B$ insulators is to insure that neighboring $c i s$-regulatory domains function autonomously. For example, deletion of the Fab-7 insulator fuses the $i a b-6$ and iab-7- domains [90]. The cross talk between silencers and activators in the two domains results in the inappropriate specification of abdominal parasegment PS11 by the fused domain. When other insulators, like $s c s$ or $s u(H w)$ are substituted for Fab-7, they block cross talk between the two $i a b$ cis-regulatory domains (though for $s u(H w)$ this activity is tissue specific and is lost in the CNS) [91]. However, these heterologous insulators don't provide the bypass or promoter interaction functions of $\mathrm{Fab}-7$ and instead block the $i a b-5$ and $i a b-6$ domains from regulating $A b d-B$. Of course as can be seen in Fig. 5, the arrangement of $c i s$-regulatory domains in the Abd-B TARD requires that the Fab- 6 and Fab- 8 insulators must also be able to support bypass and promoter interactions.

The bypass and promoter interaction activities of the three $A b d-B$ TARD insulators in BX-C are recapitulated in transgene assays. Fab-7 supports bypass and interactions with the $A b d-B$ promoter when paired with $F a b-6$ and $F a b-8$. However, Fab-7 doesn't support bypass when paired with $M c p$, or with either of the other $a b d-A$ TARD insulators, Fab-3 and Fab-4 $[65,92]$. This failure to interact in bypass/promoter targeting assays could, in principle, provide a mechanism for physically and functionally separating the $A b d-B$ TARD from the $a b d-A$ TARD. This separation would insure that different $c i s$-regulatory domains within each TARD are not only insulated from each other, but are also correctly targeted to their respective homeotic genes. Although equivalent studies haven't been done for the insulators in the $U b x$ TARD, a plausible speculation is that they will also generate an independent or semi-independent unit allowing both insulation and bypass within the Ubx TARD, while not supporting cross-regulatory interactions with the adjacent $a b d-A$ or $A b d-B$ TARDs.

Seemingly consistent with the idea that functional units in BX-C are three TARDs, the $U b x$ gene in Drosophila virilis is included in the Ant complex instead of BX-C. The breakpoint that separates virilis $U b x$ from $a b d-A / A b d-B$ maps to the insulator in melanogaster that marks the border between the $U b x$ and $a b d-A$ TARDs [93]. Since this rearrangement conserves the basic TARDs organization, the patterns of $U b x$ and $a b d-A$ expression are similar in the two species. However, one would imagine that an important mechanism for evolving new traits or forms would be chromosomal rearrangements that disrupt or alter TARD configurations by introducing or removing insulators or by reshuffling their order relative to regulatory elements and their target genes [see for example, 94].

\section{TARDs and TADs}

3C experiments in Drosophila and vertebrates have provided compelling evidence that chromosomes are subdivided into a series of Topologically Associated Domains or TADs 
[for review see 95]. Drosophila TADs are 10-500 kb with a median length of $\sim 100 \mathrm{~kb}$ [96]. The TADs in humans and mice range from $\sim 100 \mathrm{~kb}$ to several MBs with a median length of 1MB [97,98]. Multiple long-range associations are observed for sequences within each $\mathrm{TAD}$, while contacts between sequences in adjacent TADs are considerably less frequent. Consistent with the idea that they represent units of genetic regulation, TADs are often aligned with active and repressive epigenetic marks [96,97]. Moreover, expression profiles of genes within the same TAD tend to be correlated with each other [98]. As is the case for regulatory interactions within the three BX-C TARDs, long distance physical contacts between enhancers and promoters within a TAD often bypass one or more putative insulators (as defined by CTCF association)[66]. While these findings have been interpreted as evidence that a substantial fraction of the CTCF sites within a TAD might not function as "classical" insulators, an alternative view is that cross-CTCF interactions arise by bypass and/or promoter targeting mechanisms similar to those operating within but usually not between the three BX-C TARDs.

TADs are typically separated from each other by distinct "boundaries" that are often enriched for insulator binding sites [96,97]. Since interactions between elements [enhancers, promoters or insulators] on opposite sides of a TAD boundary occur infrequently an important question is what happens to chromosome topology and gene expression when a boundary is deleted. Nora et al [98] examined the consequences of deleting a TAD boundary on the $\mathrm{X}$ chromosome that overlaps the $\mathrm{X}$-inactivation center. Two effects were evident. First, the two TADs merged and many new contacts were established between elements located to either side of the deleted boundary. However, the merger wasn't complete, and elements located farther from the deleted boundary tended to retain their normal set of contacts. Second, the expression profiles of genes within the flanking TADs were altered.

At this point it isn't entirely certain what distinguishes a CTCF containing TAD boundary from the CTCF associated elements that are located within a TAD. One idea is that TAD boundaries might be special in that they can function autonomously or semi-autonomously, while the internal CTCF elements might resemble non-autonomous fly insulators and depend upon the identity of other insulators (or interacting elements) in their neighborhood. Another idea is that TAD boundaries are also non-autonomous, but preferentially pair with elements located within one TAD as opposed to the other as is seen for the BX-C TARDS. A similar, though less pronounced "self" preference for elements within a TAD would then explain the incomplete merger of the TADs flanking the deleted boundary.

Interestingly, Nora et al [98] found that while the arrangement of TADs along the chromosome is generally conserved between embryonic stem cells [ESCs], neuronal progenitor cells [NPCs] and mouse embryonic fibroblasts [MEFs], changes in the organization of contact loops within a TAD were observed. An even more dramatic example of differentiation dependent changes in looping patterns is observed for the Hox genes in the center of the mouse HoxD complex in developing limb [99]. During the early stages of limb development these central Hox genes make contact and are activated in the progenitors of the arm and forearm by cis-regulatory elements located in a "telomeric" TAD that extends from the Hox complex towards the telomere. Late in development, in the progenitors of the 
paw, the central Hox genes contact and are activated by cis-regulatory elements located in a different "centromeric" TAD that extends from the HoxD complex towards the centromere.

One potential mechanism for changing the organization of loops in (or between) TADs would be to alter the activity of insulators within these large domains. Consistent with this idea, cell type specific differences in the distribution of insulator proteins have been observed [24, 25, 100]. For vertebrate CTCF, for example, binding can be controlled by DNA methylation or nucleosome position $[50,51,100]$, while activity can be regulated by post-translational modifications and changing potential partners [101-4]. However, if CTCF were the only DNA binding protein with insulator activity in vertebrates, it is not clear that these forms of regulation would be sufficient to generate the specific/preferential long distance interactions evident within or between TADs. Studies in flies argue that interaction specificity is generated through the deployment of different combinations and arrangements of DNA binding and accessory proteins at each insulator. Thus, a reasonable expectation is that vertebrates will also depend upon other broadly expressed insulator proteins to generate the level of specificity evident in $3 \mathrm{C}$ experiments. The studies implicating the GAF ortholog, Th-P0K in Hox complex insulators would fit with this idea [58]. As for the differences in the organization of contact loops in different cell types, this could be achieved by deploying insulator proteins whose expression is limited to specific stages of development or tissues. As in flies, members of the mammalian BEN domain family would be especially good candidates for coupling insulator activity to development and differentiation.

\section{Conclusions and outlook}

New insights into the functioning and diverse activities of insulators, together with the discovery that their activities can be subject to stage and or tissue specific regulation requires us to rethink their roles both in the domain/sub-domain organization of eukaryotic chromosomes and in defining and re-defining gene regulatory networks. Several lines of evidence indicate that insulators in flies (and likely also in vertebrates) do not function autonomously. Instead, their activities in subdividing the chromosome and in gene regulation require and are determine by interactions with other insulators in their neighborhood. In addition, while it was previously thought that insulators function only to restrict the scope of action of enhancers/silencers, we now understand that insulators can also promote (by insulator bypass or by contacting promoters) long distance interactions between these regulatory elements and target genes. Finally, the discovery of a class of insulator proteins whose activity is developmentally restricted suggests that the activity of insulators, and consequently the organization of gene regulatory networks will be subject to stage and tissue specific regulation. It seems clear that future progress in understanding how regulatory networks are defined and re-defined during differentiation and development will depend upon being able to decipher the rules that govern and lend specificity to insulator interactions.

\section{Acknowledgments}

The authors would like to acknowledge support from US National Institutes of Health (GM043432) and from the Ministry of Education and Science of the Russian Federation (14.B25.31.0022) to P.S., from the RFBR 
(12-04-00195-a to D.C., 11-04-01250-a to M.E.), from the RFBR (12-04-92423-EMBL-a, 13-04-93106-CNRS_a) and from the Ministry of Education and Science of the Russian Federation (project no. 8103) to P.G.

\section{References}

1. Welshons WJ, Keppy DO. Intragenic deletions and salivary gland relationships in Drosophila. Genetics. 1975; 80:143-55. [PubMed: 805754]

2. Vazquez J, Schedl P. Deletion of an insulator element by the mutation facet-strawberry in Drosophila melanogaster. Genetics. 2000; 155:1297-311. [PubMed: 10880489]

3. Vogelmann J, Valeri A, Guillou E, Cuvier O, Nollmann M. Roles of chromatin insulator proteins in higher-order chromatin organization and transcription regulation. Nucleus. 2011; 2:358-69. [PubMed: 21983085]

4. Kirkland JG, Raab JR, Kamakaka RT. TFIIIC bound DNA elements in nuclear organization and insulation. Biochim Biophys Acta. 2013; 1829:418-24. [PubMed: 23000638]

5. Kim TH, Abdullaev ZK, Smith AD, Ching KA, et al. Analysis of the vertebrate insulator protein CTCF-binding sites in the human genome. Cell. 2007; 128:1231-45. [PubMed: 17382889]

6. Cuddapah S, Jothi R, Schones DE, Roh TY, Cui K, Zhao K. Global analysis of the insulator binding protein CTCF in chromatin barrier regions reveals demarcation of active and repressive domains. Genome Res. 2009; 19:24-32. [PubMed: 19056695]

7. Nègre N, Brown CD, Shah PK, Kheradpour P, et al. A comprehensive map of insulator elements for the Drosophila genome. PLoS Genet. 2010; 6:e1000814. [PubMed: 20084099]

8. Bell AC, West AG, Felsenfeld G. The protein CTCF is required for the enhancer blocking activity of vertebrate insulators. Cell. 1999; 98:387-96. [PubMed: 10458613]

9. Harrison DA, Gdula DA, Coyne RS, Corces VG. A leucine zipper domain of the suppressor of Hairy-wing protein mediates its repressive effect on enhancer function. Genes Dev. 1993; 7:196678. [PubMed: 7916729]

10. Kim J, Shen B, Rosen C, Dorsett D. The DNA-binding and enhancer-blocking domains of the Drosophila suppressor of Hairy-wing protein. Mol Cell Biol. 1996; 16:3381-92. [PubMed: 8668153]

11. Moon H, Filippova G, Loukinov D, Pugacheva E, et al. CTCF is conserved from Drosophila to humans and confers enhancer blocking of the Fab-8 insulator. EMBO Rep. 2005; 6:165-70. [PubMed: 15678159]

12. Gaszner M, Vazquez J, Schedl P. The Zw5 protein, a component of the scs chromatin domain boundary, is able to block enhancer-promoter interaction. Genes Dev. 1999; 13:2098-107. [PubMed: 10465787]

13. Schweinsberg S, Hagstrom K, Gohl D, Schedl P, et al. The enhancer-blocking activity of the Fab-7 boundary from the Drosophila bithorax complex requires GAGA-factor-binding sites. Genetics. 2004; 168:1371-84. [PubMed: 15579691]

14. Belozerov VE, Majumder P, Shen P, Cai HN. A novel boundary element may facilitate independent gene regulation in the Antennapedia complex of Drosophila. EMBO J. 2003; 22:3113-21. [PubMed: 12805225]

15. Zhao K, Hart CM, Laemmli UK. Visualization of chromosomal domains with boundary elementassociated factor BEAF-32. Cell. 1995; 81:879-89. [PubMed: 7781065]

16. Aoki T, Sarkeshik A, Yates J, Schedl P. Elba, a novel developmentally regulated chromatin boundary factor is a hetero-tripartite DNA binding complex. Elife. 2012; 1:e00171. [PubMed: 23240086]

17. Scott KS, Taubman AD, Geyer PK. Enhancer blocking by the Drosophila gypsy insulator depends upon insulator anatomy and enhancer strength. Genetics. 1999; 153:787-98. [PubMed: 10511558]

18. Golovnin A, Biryukova I, Romanova O, Silicheva M, et al. An endogenous $\mathrm{Su}(\mathrm{Hw})$ insulator separates the yellow gene from the Achaete-scute gene complex in Drosophila. Development. 2003; 130:3249-58. [PubMed: 12783795]

19. Parnell TJ, Viering MM, Skjesol A, Helou C, et al. An endogenous suppressor of hairy-wing insulator separates regulatory domains in Drosophila. Proc Natl Acad Sci U S A. 2003; 100:13436-41. [PubMed: 14597701] 
20. Soshnev AA, Li X, Wehling MD, Geyer PK. Context differences reveal insulator and activator functions of a Su(Hw) binding region. PLoS Genet. 2008; 4:e1000159. [PubMed: 18704163]

21. Smith ST, Wickramasinghe P, Olson A, Loukinov D, et al. Genome wide ChIP-chip analyses reveal important roles for CTCF in Drosophila genome organization. Dev Biol. 2009; 328:518-28. [PubMed: 19210964]

22. Bartkuhn M, Straub T, Herold M, Herrmann M, et al. Active promoters and insulators are marked by the centrosomal protein 190. EMBO J. 2009; 28:877-88. [PubMed: 19229299]

23. Jiang N, Emberly E, Cuvier O, Hart CM. Genome-wide mapping of boundary element-associated factor (BEAF) binding sites in Drosophila melanogaster links BEAF to transcription. Mol Cell Biol. 2009; 29:3556-68. [PubMed: 19380483]

24. Bushey AM, Ramos E, Corces VG. Three subclasses of a Drosophila insulator show distinct and cell type-specific genomic distributions. Genes Dev. 2009; 23:1338-50. [PubMed: 19443682]

25. Nègre N, Brown CD, Ma L, Bristow CA, et al. A cis-regulatory map of the Drosophila genome. Nature. 2011; 471:527-31. [PubMed: 21430782]

26. Schwartz YB, Linder-Basso D, Kharchenko PV, Tolstorukov MY, et al. Nature and function of insulator protein binding sites in the Drosophila genome. Genome Res. 2012; 22:2188-98. [PubMed: 22767387]

27. Fujioka M, Wu X, Jaynes JB. A chromatin insulator mediates transgene homing and very longrange enhancer-promoter communication. Development. 2009; 136:3077-87. [PubMed: 19675129]

28. Parkhurst SM, Harrison DA, Remington MP, Spana C, et al. The Drosophila su[Hw] gene, which controls the phenotypic effect of the gypsy transposable element, encodes a putative DNA-binding protein. Genes Dev. 1988; 2:1205-15. [PubMed: 2462523]

29. Büchner K, Roth P, Schotta G, Krauss V, et al. Genetic and molecular complexity of the position effect variegation modifier mod(mdg4) in Drosophila. Genetics. 2000; 155:141-57. [PubMed: 10790390]

30. Gause M, Morcillo P, Dorsett D. Insulation of enhancer-promoter communication by a gypsy transposon insert in the Drosophila cut gene: cooperation between suppressor of hairy-wing and modifier of mdg4 proteins. Mol Cell Biol. 2001; 21:4807-17. [PubMed: 11416154]

31. Ghosh D, Gerasimova TI, Corces VG. Interactions between the $\mathrm{Su}(\mathrm{Hw})$ and $\mathrm{Mod}(\mathrm{mdg} 4)$ proteins required for gypsy insulator function. EMBO J. 2001; 20:2518-27. [PubMed: 11350941]

32. Pai CY, Lei EP, Ghosh D, Corces VG. The centrosomal protein CP190 is a component of the gypsy chromatin insulator. Mol Cell. 2004; 16:737-48. [PubMed: 15574329]

33. Golovnin A, Mazur A, Kopantseva M, Kurshakova M, et al. Integrity of the Mod(mdg4)-67.2 BTB domain is critical to insulator function in Drosophila melanogaster. Mol Cell Biol. 2007; 27:96374. [PubMed: 17101769]

34. Bonchuk A, Denisov S, Georgiev P, Maksimenko O. Drosophila BTB/POZ domains of "ttk group" can form multimers and selectively interact with each other. J Mol Biol. 2011; 412:423-36. [PubMed: 21821048]

35. Kurshakova M, Maksimenko O, Golovnin A, Pulina M, et al. Evolutionarily conserved E(y)2/Sus1 protein is essential for the barrier activity of $\mathrm{Su}(\mathrm{Hw})$-dependent insulators in Drosophila. Mol Cell. 2007; 27:332-8. [PubMed: 17643381]

36. Herold M, Bartkuhn M, Renkawitz R. CTCF: insights into insulator function during development. Development. 2012; 139:1045-57. [PubMed: 22354838]

37. Chung JH, Whiteley M, Felsenfeld G. A $5^{\prime}$ element of the chicken beta-globin domain serves as an insulator in human erythroid cells and protects against position effect in Drosophila. Cell. 1993; 74:505-14. [PubMed: 8348617]

38. Xiao T, Wallace J, Felsenfeld G. Specific sites in the C terminus of CTCF interact with the SA2 subunit of the cohesin complex and are required for cohesin-dependent insulation activity. Mol Cell Biol. 2011; 31:2174-83. [PubMed: 21444719]

39. Mohan M, Bartkuhn M, Herold M, Philippen A, et al. The Drosophila insulator proteins CTCF and CP190 link enhancer blocking to body patterning. EMBO J. 2007; 26:4203-14. [PubMed: 17805343] 
40. Gerasimova TI, Lei EP, Bushey AM, Corces VG. Coordinated control of dCTCF and gypsy chromatin insulators in Drosophila. Mol Cell. 2007; 28:761-72. [PubMed: 18082602]

41. Espinás ML, Jiménez-García E, Vaquero A, Canudas S, et al. The N-terminal POZ domain of GAGA mediates the formation of oligomers that bind DNA with high affinity and specificity. $\mathrm{J}$ Biol Chem. 1999; 274:16461-9. [PubMed: 10347208]

42. Melnikova L, Juge F, Gruzdeva N, Mazur A, et al. Interaction between the GAGA factor and Mod(mdg4) proteins promotes insulator bypass in Drosophila. Proc Natl Acad Sci U S A. 2004; 101:14806-11. [PubMed: 15465920]

43. Bonchuk A, Denisov S, Georgiev P, Maksimenko O. Drosophila BTB/POZ domains of "ttk group" can form multimers and selectively interact with each other. J Mol Biol. 2011; 412:423-36. [PubMed: 21821048]

44. Soeller WC, Poole SJ, Kornberg T. In vitro transcription of the Drosophila engrailed gene. Genes Dev. 1988; 2:68-81. [PubMed: 3356339]

45. Katsani KR, Hajibagheri MA, Verrijzer CP. Co-operative DNA binding by GAGA transcription factor requires the conserved $\mathrm{BTB} / \mathrm{POZ}$ domain and reorganizes promoter topology. EMBO J. 1999; 18:698-708. [PubMed: 9927429]

46. Tsukiyama T, Becker PB, Wu C. ATP-dependent nucleosome disruption at a heat-shock promoter mediated by binding of GAGA transcription factor. Nature. 1994; 367:525-32. [PubMed: 8107823]

47. Wall G, Varga-Weisz PD, Sandaltzopoulos R, Becker PB. Chromatin remodeling by GAGA factor and heat shock factor at the hypersensitive Drosophila hsp26 promoter in vitro. EMBO J. 1995; 14:1727-36. [PubMed: 7737124]

48. Li J, Gilmour DS. Distinct mechanisms of transcriptional pausing orchestrated by GAGA factor and M1BP, a novel transcription factor. EMBO J. 2013; 32:1829-41. [PubMed: 23708796]

49. Mishra K, Chopra VS, Srinivasan A, Mishra RK. Trl-GAGA directly interacts with lola like and both are part of the repressive complex of Polycomb group of genes. Mech Dev. 2003; 120:681-9. [PubMed: 12834867]

50. Bell AC, Felsenfeld G. Methylation of a CTCF-dependent boundary controls imprinted expression of the Igf2 gene. Nature. 2000; 405:482-5. [PubMed: 10839546]

51. Hark AT, Schoenherr CJ, Katz DJ, Ingram RS, et al. CTCF mediates methylation-sensitive enhancer-blocking activity at the H19/Igf2 locus. Nature. 2000; 405:486-9. [PubMed: 10839547]

52. Abhiman S, Iyer LM, Aravind L. BEN: a novel domain in chromatin factors and DNA viral proteins. Bioinformatics. 2008; 24:458-61. [PubMed: 18203771]

53. Dai Q, Ren A, Westholm JO, Serganov AA, et al. The BEN domain is a novel sequence-specific DNA-binding domain conserved in neural transcriptional repressors. Genes Dev. 2013; 27:60214T. [PubMed: 23468431]

54. Duan H, Dai Q, Kavaler J, Bejarano F, et al. Insensitive is a corepressor for Suppressor of Hairless and regulates Notch signalling during neural development. EMBO J. 2011; 30:3120-33. [PubMed: 21765394]

55. Dai Q, Andreu-Agullo C, Insolera R, Wong LC, et al. BEND6 is a nuclear antagonist of Notch signaling during self-renewal of neural stem cells. Development. 2013; 140:1892-902. [PubMed: 23571214]

56. Nakayama K, Nakayama N, Davidson B, Sheu JJ, et al. A BTB/POZ protein, NAC-1, is related to tumor recurrence and is essential for tumor growth and survival. Proc Natl Acad Sci U S A. 2006; 103:18739-44. [PubMed: 17130457]

57. Wang J, Rao S, Chu J, Shen X, et al. A protein interaction network for pluripotency of embryonic stem cells. Nature. 2006; 444:364-8. [PubMed: 17093407]

58. Srivastava S, Puri D, Garapati HS, Dhawan J, Mishra RK. Vertebrate GAGA factor associated insulator elements demarcate homeotic genes in the HOX clusters. Epigenetics Chromatin. 2013; 6:8. [PubMed: 23607454]

59. Muravyova E, Golovnin A, Gracheva E, Parshikov A, et al. Loss of insulator activity by paired $\mathrm{Su}(\mathrm{Hw})$ chromatin insulators. Science. 2001; 291:495-8. [PubMed: 11161206]

60. Cai HN, Shen P. Effects of cis arrangement of chromatin insulators on enhancer-blocking activity. Science. 2001; 291:493-5. [PubMed: 11161205] 
61. Comet I, Savitskaya E, Schuettengruber B, Nègre N, et al. PRE-mediated bypass of two Su(Hw) insulators targets PcG proteins to a downstream promoter. Dev Cell. 2006; 11:117-24. [PubMed: 16824958]

62. Conte C, Dastugue B, Vaury C. Coupling of enhancer and insulator properties identified in two retrotransposons modulates their mutagenic impact on nearby genes. Mol Cell Biol. 2002; 22:1767-77. [PubMed: 11865056]

63. Kuhn EJ, Viering MM, Rhodes KM, Geyer PK. A test of insulator interactions in Drosophila. EMBO J. 2003; 22:2463-71. [PubMed: 12743040]

64. Gruzdeva N, Kyrchanova O, Parshikov A, Kullyev A, Georgiev P. The Mcp element from the bithorax complex contains an insulator that is capable of pairwise interactions and can facilitate enhancer-promoter communication. Mol Cell Biol. 2005; 25:3682-9. [PubMed: 15831473]

65. Kyrchanova O, Chetverina D, Maksimenko O, Kullyev A, Georgiev P. Orientation-dependent interaction between Drosophila insulators is a property of this class of regulatory elements. Nucleic Acids Res. 2008; 36:7019-28. [PubMed: 18987002]

66. Sanyal A, Lajoie BR, Jain G, Dekker J. The long-range interaction landscape of gene promoters. Nature. 2012; 489:109-13. [PubMed: 22955621]

67. Blanton J, Gaszner M, Schedl P. Protein:protein interactions and the pairing of boundary elements in vivo. Genes Dev. 2003; 17:664-75. [PubMed: 12629048]

68. Tolhuis B, Palstra RJ, Splinter E, Grosveld F, de Laat W. Looping and interaction between hypersensitive sites in the active beta-globin locus. Mol Cell. 2002; 10:1453-65. [PubMed: 12504019]

69. Splinter E, Heath H, Kooren J, Palstra RJ, et al. CTCF mediates long-range chromatin looping and local histone modification in the beta-globin locus. Genes Dev. 2006; 20:2349-54. [PubMed: 16951251]

70. Handoko L, Xu H, Li G, Ngan CY, et al. CTCF-mediated functional chromatin interactome in pluripotent cells. Nat Genet. 2011; 43:630-8. [PubMed: 21685913]

71. Hadjur S, Williams LM, Ryan NK, Cobb BS, et al. Cohesins form chromosomal cis-interactions at the developmentally regulated IFNG locus. Nature. 2009; 460:410-3. [PubMed: 19458616]

72. Nativio R, Wendt KS, Ito Y, Huddleston JE, et al. Cohesin is required for higher-order chromatin conformation at the imprinted IGF2-H19 locus. PLoS Genet. 2009; 5:e1000739. [PubMed: 19956766]

73. Chien R, Zeng W, Kawauchi S, Bender MA, et al. Cohesin mediates chromatin interactions that regulate mammalian $\beta$-globin expression. J Biol Chem. 2011; 286:17870-8. [PubMed: 21454523]

74. Muller M, Hagstrom K, Gyurkovics H, Pirrotta V, Schedl P. The Mcp element from the Drosophila melanogaster bithorax complex mediates long-distance regulatory interactions. Genetics. 1999; 153:1333-56. [PubMed: 10545463]

75. Vazquez J, Müller M, Pirrotta V, Sedat JW. The Mcp element mediates stable long-range chromosome-chromosome interactions in Drosophila. Mol Biol Cell. 2006; 17:2158-65. [PubMed: 16495335]

76. Kravchenko E, Savitskaya E, Kravchuk O, Parshikov A, et al. Pairing between gypsy insulators facilitates the enhancer action in trans throughout the Drosophila genome. Mol Cell Biol. 2005; 25:9283-91. [PubMed: 16227580]

77. Cléard F, Moshkin Y, Karch F, Maeda RK. Probing long-distance regulatory interactions in the Drosophila melanogaster bithorax complex using Dam identification. Nat Genet. 2006; 38:931-5. [PubMed: 16823379]

78. Li HB, Müller M, Bahechar IA, Kyrchanova O, et al. Insulators, not Polycomb response elements, are required for long-range interactions between Polycomb targets in Drosophila melanogaster. Mol Cell Biol. 2011; 31:616-25. [PubMed: 21135119]

79. Gohl D, Aoki T, Blanton J, Shanower G, et al. Mechanism of chromosomal boundary action: roadblock, sink, or loop? Genetics. 2011; 187:731-48. [PubMed: 21196526]

80. Majumder P, Cai HN. The functional analysis of insulator interactions in the Drosophila embryo. Proc Natl Acad Sci U S A. 2003; 100:5223-8. [PubMed: 12700350]

81. Rodin S, Kyrchanova O, Pomerantseva E, Parshikov A, Georgiev P. New properties of Drosophila Fab-7 insulator. Genetics. 2007; 177:113-21. [PubMed: 17890362] 
82. Kyrchanova O, Toshchakov S, Podstreshnaya Y, Parshikov A, Georgiev P. Functional interaction between the Fab-7 and Fab- 8 boundaries and the upstream promoter region in the Drosophila AbdB gene. Mol Cell Biol. 2008; 28:4188-95. [PubMed: 18426914]

83. Kyrchanova O, Toshchakov S, Parshikov A, Georgiev P. Study of the functional interaction between Mcp insulators from the Drosophila bithorax complex: effects of insulator pairing on enhancer-promoter communication. Mol Cell Biol. 2007; 27:3035-43. [PubMed: 17283051]

84. Krivega M, Savitskaya E, Krivega I, Karakozova M, et al. Interaction between a pair of gypsy insulators or between heterologous gypsy and Wari insulators modulates Flp site-specific recombination in Drosophila melanogaster. Chromosoma. 2010; 119:425-34. [PubMed: 20354861]

85. Palstra RJ, Tolhuis B, Splinter E, Nijmeijer R, et al. The beta-globin nuclear compartment in development and erythroid differentiation. Nat Genet. 2003; 35:190-4. [PubMed: 14517543]

86. Li T, Hu JF, Qiu X, Ling J, et al. CTCF regulates allelic expression of Igf2 by orchestrating a promoter-polycomb repressive complex 2 intrachromosomal loop. Mol Cell Biol. 2008; 28:647382. [PubMed: 18662993]

87. Erokhin M, Davydova A, Kyrchanova O, Parshikov A, et al. Insulators form gene loops by interacting with promoters in Drosophila. Development. 2011; 138:4097-106. [PubMed: 21862564]

88. Liu Z, Scannell DR, Eisen MB, Tjian R. Control of embryonic stem cell lineage commitment by core promoter factor, TAF3. Cell. 2011; 146:720-31. [PubMed: 21884934]

89. Maeda RK, Karch F. The bithorax complex of Drosophila an exceptional Hox cluster. Curr Top Dev Biol. 2009; 88:1-33. [PubMed: 19651300]

90. Gyurkovics H, Gausz J, Kummer J, Karch F. A new homeotic mutation in the Drosophila bithorax complex removes a boundary separating two domains of regulation. EMBO J. 1990; 9:2579-85. [PubMed: 1973385]

91. Hogga I, Mihaly J, Barges S, Karch F. Replacement of Fab-7 by the gypsy or scs insulator disrupts long-distance regulatory interactions in the Abd-B gene of the bithorax complex. Mol Cell. 2001; 8:1145-51. [PubMed: 11741549]

92. Kyrchanova O, Ivlieva T, Toshchakov S, Parshikov A, et al. Selective interactions of boundaries with upstream region of Abd-B promoter in Drosophila bithorax complex and role of dCTCF in this process. Nucleic Acids Res. 2011; 39:3042-52. [PubMed: 21149269]

93. Bender W, Lucas M. The border between the ultrabithorax and abdominal-A regulatory domains in the drosophila bithorax complex. Genetics. 2013; 193:1135-47. [PubMed: 23288934]

94. Cande JD, Chopra VS, Levine M. Evolving enhancer-promoter interactions within the tinman complex of the flour beetle, Tribolium castaneum. Development. 2009; 136:3153-60. [PubMed: 19700619]

95. Nora EP, Dekker J, Heard E. Segmental folding of chromosomes: A basis for structural and regulatory chromosomal neighborhoods? Bioessays. 2013 Jul 5.10.1002/bies.201300040

96. Sexton T, Yaffe E, Kenigsberg E, Bantignies F, et al. Three-dimensional folding and functional organization principles of the Drosophila genome. Cell. 2012; 148:458-72. [PubMed: 22265598]

97. Dixon JR, Selvaraj S, Yue F, Kim A, et al. Topological domains in mammalian genomes identified by analysis of chromatin interactions. Nature. 2012; 485:376-80. [PubMed: 22495300]

98. Nora EP, Lajoie BR, Schulz EG, Giorgetti L, et al. Spatial partitioning of the regulatory landscape of the X-inactivation centre. Nature. 2012; 485:381-5. [PubMed: 22495304]

99. Andrey G, Montavon T, Mascrez B, Gonzalez F, et al. A switch between topological domains underlies HoxD genes collinearity in mouse limbs. Science. 2013; 340:1234167. doi:10.1126. [PubMed: 23744951]

100. Wang H, Maurano MT, Qu H, Varley KE, et al. Widespread plasticity in CTCF occupancy linked to DNA methylation. Genome Res. 2012; 22:1680-8. [PubMed: 22955980]

101. Klenova EM, Chernukhin IV, El-Kady A, Lee RE, et al. Functional phosphorylation sites in the C-terminal region of the multivalent multifunctional transcriptional factor CTCF. Mol Cell Biol. 2001; 21:2221-34. [PubMed: 11238955] Yu W, Ginjala V, Pant V, Chernukhin I, et al. Poly(ADP-ribosyl)ation regulates CTCF-dependent chromatin insulation. Nat Genet. 36:110510. [PubMed: 15361875] 
102. Farrar D, Rai S, Chernukhin I, Jagodic M, et al. Mutational analysis of the poly(ADPribosyl)ation sites of the transcription factor CTCF provides an insight into the mechanism of its regulation by poly(ADP-ribosyl)ation. Mol Cell Biol. 2010; 30:1199-216. [PubMed: 20038529]

103. MacPherson MJ, Beatty LG, Zhou W, Du M, Sadowski PD. The CTCF insulator protein is posttranslationally modified by SUMO. Mol Cell Biol. 2009; 29:714-25. [PubMed: 19029252]

104. Kitchen NS, Schoenherr CJ. Sumoylation modulates a domain in CTCF that activates transcription and decondenses chromatin. J Cell Biochem. 2010; 111:665-75. [PubMed: 20589758] 

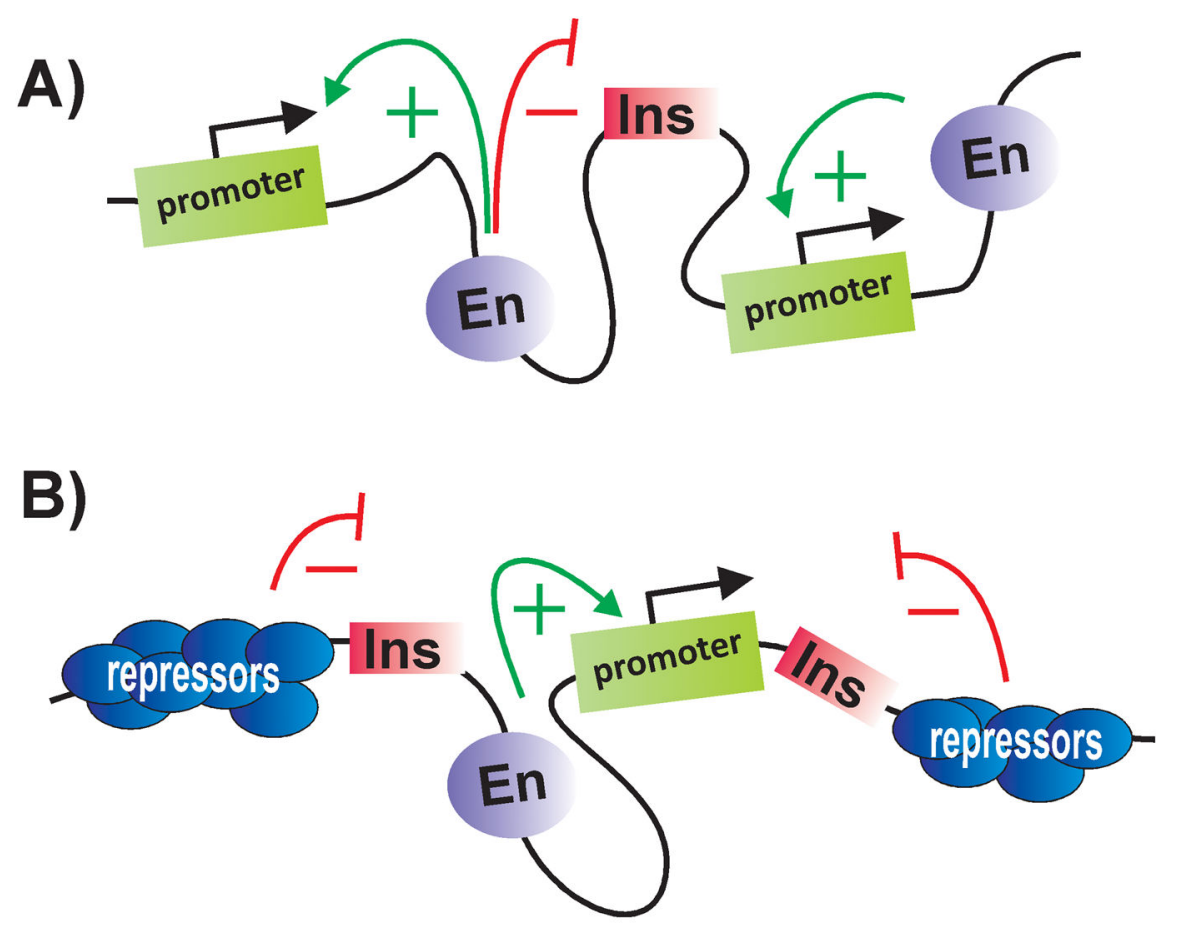

Figure 1. Classical assays for insulator activity

A: Enhancer-blocking activity. Insulators function in a position-dependent manner, interfering with the activity of enhancers (or silencers) only when inserted between an enhancer (silencer) and the promoter. B: Barrier activity. Insulators bracketing a reporter can protect against the encroachment heterochromatic repression from the chromatin flanking the transgene insertion site. En enhancer; Ins insulator. Arrows mark the promoter regions of the genes. 
A)

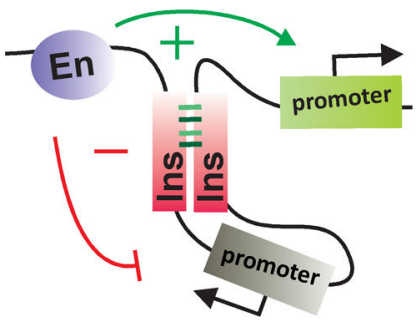

B)

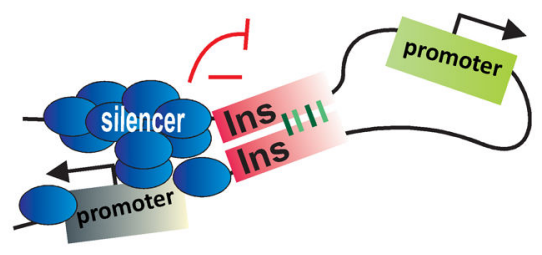

Figure 2. Insulators are bypassed by insulator pairing

When two insulators are inserted between an enhancer and a reporter, the insulators are "bypassed" by a mechanism that depends upon insulator:insulator interactions. A: Insulator pairing loops out the intervening DNA, bringing the enhancer in close proximity to a gene (green) located immediately downstream of the paired insulators. While the enhancer activates this gene, the gene within the loop (grey) is insulated from the enhancer. B: By a similar mechanism, insulator pairing can bring a distant silencer in contact with a promoter, repressing rather than activating the transcription of this gene (grey). In this instance, the gene (green) located within the loop between the paired insulators is protected against silencing. 

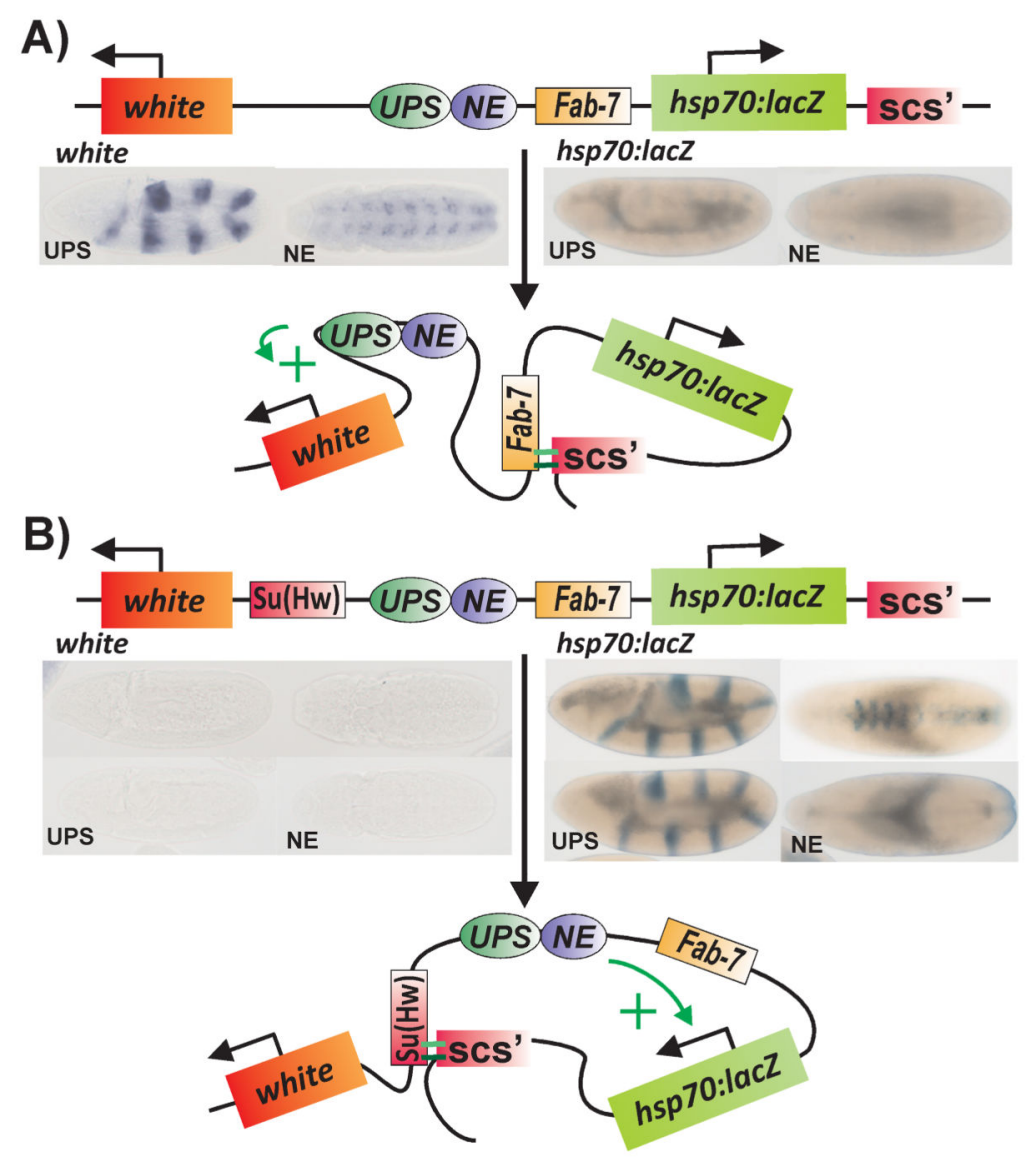

Figure 3. Domain definition: Insulators define regulatory domains through preferential interactions with their neighbors

A: Interposing the Fab-7 insulator between the fushi taratzu ( $f t z$ ) stripe (UPS) and neurogenic (NE) enhancers and the hsp70:lacZ reporter subdivides the transgene into two independent regulatory domains. Since white and the $\mathrm{ftz}$ enhancers are in the same domain, the two enhancers activate white expression in a stripe pattern in the early embryo, and in the CNS during mid-embryogenesis. By contrast, the hsp70:lacZ reporter is in a domain defined by $\mathrm{Fab}-7$ and the scs' insulator located downstream of the lacZ gene. As a consequence, the $l a c Z$ reporter is insulated from the $f t z$ enhancers. B: The regulatory domains in the transgene are redefined when $\mathrm{Fab}-7$ is challenged by introducing a $3^{\text {rd }}$ insulator, $s u(H w)$, between white and the $f t z$ enhancers. Embryo on top: As shown in the illustration, white is in a domain by itself, while the ftz enhancers, Fab-7 and the hsp70:lacZ reporters are in the same domain. In this configuration, Fab-7 is bypassed and the $\mathrm{ftz}$ enhancers activate lac $Z$ expression in a stripe pattern in early embryos, and subsequently in the CNS during mid-embryogenesis. Embryo on the bottom: For this transgene insert, the domain pattern changes between early and mid-embryogenesis. During early embryogenesis, the UPS enhancer, $F a b-7$ and $h s p 70: l a c Z$ are in the same regulatory domain and $l a c Z$ is expressed in a stripe pattern. During mid-embryogenesis, $F a b-7$ regains insulator activity and the domain configuration is redefined so that white, hsp70:lacZ and the ftz enhancers are all located in separate domains (not illustrated). Insulators: $s u(H w), F a b-7$, 
and scs'. Reporter genes: white and hsp70:lacZ. Enhancers: UPS stripe enhancer, NE CNS enhancer. 
A)

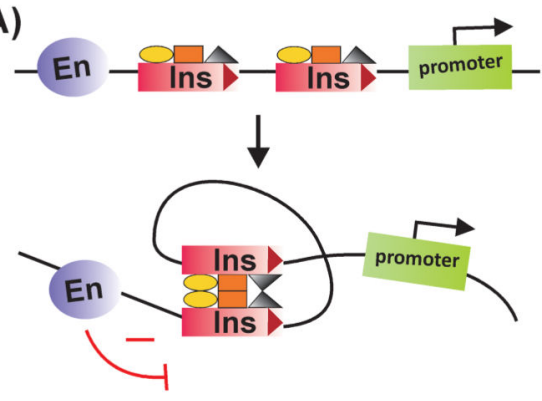

B)

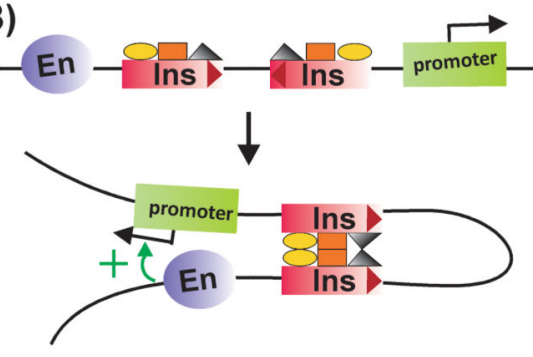

Figure 4. Insulator pairing depends upon specific, orientation dependent interactions The topology of the loop formed by pairing interactions between insulators, and thus the regulatory consequences, depends upon the orientation of the insulators respect to one another. A: In this asymmetric configuration of insulator protein binding sites, the pairing of insulators arranged in the same orientation generates a loop in which the enhancer and reporter are located far from each other. In this case no "bypass" is observed. B: In contrast, pairing of insulators arranged in opposite orientation generates a loop that brings enhancer and reporter close together. This enables the enhancer to activate transcription. 


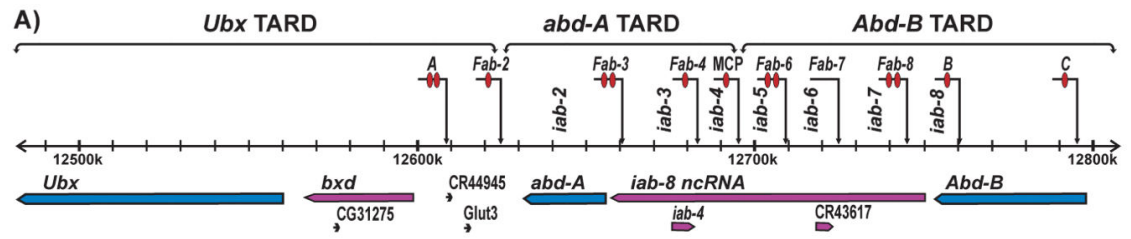

B)

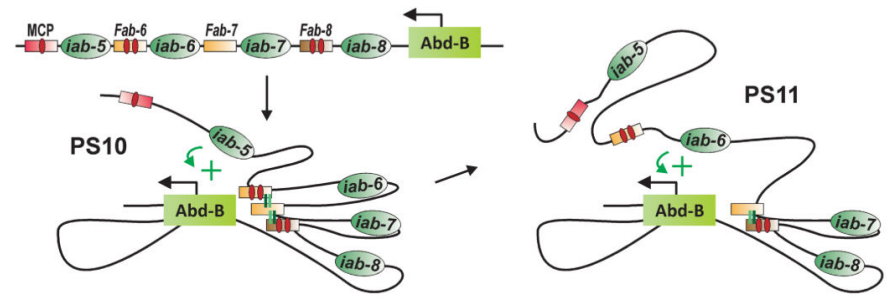

Figure 5. Insulators organize the regulatory landscape of the Drosophila bithorax complex A: Diagram of the three BX-C TARDs, containing $U b x, a b d-A$ and $A b d-B$ genes and their respective $c i s$-regulatory domains. The positions of the mapped insulator elements and $c i s-$ regulatory domains that they delimit are indicated above the sequence coordinate line. The red ovals in the insulators designate CTCF sites. B: Organization of the $A b d-B$ TARD. The $M c p$ insulator marks the boundary between the $a b d-A$ and $A b d-B$ TARDs. There are four $c i s$-regulatory domains within the $A b d-B, i a b-5, i a b-6, i a b-7$ and $i a b-8$. Located between $c i s-$ regulatory domains are the $F a b-6, F a b-7$ and $F a b-8$ insulators as indicated. In PS10 (left) the $i a b-5$ cis-regulatory domain is activated, while the $i a b-6, i a b-7$ and $i a b-8 c i s$-regulatory domains are silenced by a Polycomb dependent mechanism. The iab- 6 cis-regulatory domain bypasses the $F a b-6, F a b-7$ and $F a b-8$ insulators to activate $A b d-B$ expression in a PS10 specific pattern. In PS11 (right), both $i a b-5$ and $i a b-6$ are activated; however, the cisregulatory domain closest to the $A b d-B$ transcription unit, $i a b-6$ directs $A b d-B$ expression in a PS11 specific pattern. To do so, iab- 6 must bypass the $F a b-7$ and Fab-8 insulators in order to contact the $A b d-B$ promoter. 